Revista Destaques Acadêmicos, Lajeado, v. 11, n. 2, 2019. ISSN 2176-3070

DOI: http://dx.doi.org/10.22410/issn.2176-3070.v11i2a2019.1933

http://www.univates.br/revistas

\title{
O IMPACTO DO ENVELHECIMENTO NO ATO DE DIRIGIR E OS COMPORTAMENTOS DE AUTORREGULAÇÃO EM MOTORISTAS IDOSOS ${ }^{1}$
}

\author{
Carina Ernst ${ }^{2}$, Marta Novelo ${ }^{3}$
}

Resumo: Algumas alterações nas funções cognitivas e psicológicas presentes no envelhecimento podem afetar o ato de dirigir. Contudo, no indivíduo idoso o comportamento de autorregulação pode compensar as perdas ocorridas ao longo da vida, diante da própria percepção do declínio de suas habilidades na condução, o que, muitas vezes, culmina, finalmente, em decidir parar de dirigir completamente. Diante de um estudo teórico, este trabalho buscou explorar a relação deste cenário com os comportamentos compensatórios. Ademais, um maior entendimento sobre este assunto pode ser incorporado em programas de treinamento de motoristas mais idosos e, ainda, fornecer subsídios que auxiliem na elaboração de rodovias que reduzam os conflitos de trânsito, contribuindo, por fim, para o surgimento de tecnologias apropriadas para veículos que ajudem os condutores mais velhos a conduzir com maior segurança.

Palavras-chave: Envelhecimento. Trânsito. Autorregulação. Funções Cognitivas.

\section{INTRODUÇÃO}

A mobilidade urbana é uma temática atual, diante do avanço da globalização e do crescimento das cidades. Com isso, o trânsito adquire uma importância ímpar, já que o deslocamento com veículos terrestres é um instrumento de maior incidência: prova disso é o crescente aumento da sua frota. Em 2010, a frota circulante total era de 48,5 milhões de veículos, passando

1 Artigo acadêmico produzido no curso de Pós-Graduação Latu Senso especialização em Psicologia no Trânsito do curso de Pós-Graduação da Universidade do Vale do Taquari Univates.

2 Psicóloga, graduada em Psicologia pela Universidade de Passo Fundo - 2009 e cursando PósGraduação em Psicologia no Trânsito na Universidade do Vale do Taquari - Univates. E-mail: carina_ernst@hotmail.com.

3 Psicóloga, Mestre Gerontologia e Professora do curso de Pós-Graduação Latu Senso Especialização em Psicologia do Trânsito da Universidade do Vale do Taquari - Univates. Orientadora do trabalho. E-mail: martanovelo@gmail.com 
para 65,8 milhões em 2017. No estado do Rio Grande do Sul, a frota circulante total em 2017 ficou em 4,9 milhões, ocupando a quarta posição entre os estados, fechando o índice com 7,59\%. (IBPT, 2018).

Diante deste contexto, o motorista é alçado à condição de protagonista, o que requer do indivíduo uma conduta segura, consciente e, principalmente, responsável. Nesse cenário, surge a importante questão do crescimento da população idosa no Brasil. Com o envelhecimento acelerado da população e aumento da expectativa de vida, espera-se o acréscimo do contingente de indivíduos idosos que utilizam o meio viário. Há projeções que no ano de 2030 a população de indivíduos idosos será maior que a população infantil de 0 a 14 anos. O estado do Rio Grande do Sul detém, entre todas as unidades da federação, o maior índice de envelhecimento (IBGE). Atualmente, o número de motoristas idosos no estado com mais de 65 anos representa $12 \%$ do montante total de motoristas que possuem $\mathrm{CNH}$ ativa (http://www.detran.rs.gov.br/ lista/797/estatisticas-de-transito).

Muitos estudos demonstram que o envelhecimento causa alterações funcionais e estruturais a nível cerebral, o que, por consequência, causa um declínio em alguns processos cognitivos, mesmo diante de um envelhecimento saudável (GLYSKY, 2007). Desta forma, sugere-se que, de forma geral, motoristas idosos apresentam um desempenho prejudicado para conduzir veículos e assim um alto risco de envolver-se em acidentes (JOANNEM. WOOD, 2009; KAY et al., 2008; WATZKE; SMITH, 1994).

No entanto, mesmo que nem todos os motoristas idosos tenham um desempenho de condução prejudicado ou um alto risco de colisão, gerado pelas quedas funcionais comumente experimentadas nessa fase da vida, o risco relativo de colisão em motoristas experientes não é tão alto como poderíamos esperar (LANGFORD; KOPPEL, 2006).

Neste contexto, os comportamentos compensatórios na condução de automóveis são definidos como um processo ocorrido em adultos mais velhos para otimizar as tomadas de decisões diante dos declínios percebidos nas habilidades (DAVIS; CONLON, 2017).

Diante de um estudo teórico, este trabalho busca explorar e dirimir parte desta questão. Inclusive, um maior entendimento sobre este assunto pode ser incorporado em programas de treinamento de motoristas mais idosos. Além disso, tal compreensão pode auxiliar na estruturação de rodovias que reduzam os conflitos de trânsito, contribuindo, ainda, para a proliferação de tecnologias apropriadas para veículos, as quais ajudem os condutores mais velhos a conduzir com segurança durante mais tempo. 


\section{Funções psicológicas envolvidas no ato de dirigir e impacto do envelhecimento em condutores.}

$\mathrm{O}$ ato de dirigir envolve diversas habilidades, psicológicas, cognitivas, além de uma interação com muitos fatores externos que não podem ser controlados pelos próprios condutores. Por isso, o condutor deve estar atento ao ambiente em que está inserido, especialmente quanto às informações que surgem durante o percurso (tráfego de veículos, pedestres, avisos visuais e sonoros) e às condições adversas, tais como imprudência de outros motoristas, vias inapropriadas, condição meteorológica agressiva, trânsito intenso. A multiplicidade de fatores envolvidos, intrínsecos e ambientais, no ato de dirigir influenciam o comportamento dos indivíduos e, consequentemente, sua forma de conduzir um veículo. Desta forma, o comportamento dos condutores é apontado como uma causa relevante na ocorrência de acidentes de trânsito, sendo o fator humano considerado o principal responsável por eles (CORREIA; HORTA, 2014; GONÇALVES, 2013; MOGNON; SANTOS, 2017; PANICHI; WAGNER, 2006)

A aprendizagem veicular exige do indivíduo a manifestação de capacidades e a aquisição de habilidades motoras, sensoriais, cognitivas e de informações sobre o trânsito, com suas implicações técnicas, preventivas, defensivas e punitivas.

As funções cognitivas, quando relacionadas à direção veicular, incluem “(...) memória, atenção, avaliação sistemática do ambiente e outras habilidades visuoespaciais, verbais e de processamento de informações, tomada de decisões e resolução de problemas. Estas funções devem se processar de modo dinâmico." (Pirito, 1999, p. 27). Percebe-se, assim, que o ato de dirigir um veículo pode parecer uma atividade simples, quase automática, mas exige uma complexa articulação de funções do condutor. (BALBINOT; ZARO; TIMM, 2011).

Alguns estudos demonstram uma associação com o desempenho de condução em simuladores e o desempenho cognitivo de idosos (BUNCE et al., 2012; CASUTT et al., 2014; VASQUES et al., 2016). Em condições adversas de tempo, como chuva e neblina, que necessariamente exigem mais da capacidade de memória da cognição em geral, estão relacionadas com pior desempenho em simuladores (VASQUES et al., 2016). Por outro lado, os resultados sugerem que déficits relacionados à idade no controle executivo e de atenção podem afetar o desempenho de direção em idosos (BUNCE et al., 2012).

A função executiva é composta de processos de controle que regulam pensamentos e comportamentos, incluindo resolução de problemas, tomada de decisões, monitoramento de ações e avaliação da eficácia das ações (CHAN et al., 2008). Esta função é necessária para integrar informações e planejar uma resposta e, portanto, altamente relevante para uma direção competente, principalmente em situações de maior complexidade, como por exemplo em interseções. São nestas situações que os idosos apresentam maiores dificuldades, 
pois envolve maior ativação da capacidade de alternar de forma flexível tarefas diferentes, da capacidade de monitorar os estímulos recebidos enquanto atualiza as informações atuais e da inibição de respostas automáticas (ANSTEY et al., 2005; CHAN et al., 2008). Em conjunto com a memória de trabalho capacidade de reter e manipular informações em um curto período de tempo-, a função executiva se volta para responder a novas circunstâncias de condução e antecipar, planejar e acompanhar situações que exigem julgamento e tomada de decisão (ENGLE, 2002). No trânsito ela representa, ainda, a capacidade de retorno de faixa de domínio, a passagem pelas faixas de tráfego ou a entrada e saída de interseções (MILLER; TAYLOR-PILIAE; INSEL, 2016).

O campo funcional da atenção também ganha destaque dentro deste conjunto de fatores. A atenção, assim, pode ser definida como o fator que administra grande parte das informações adquiridas pelo indivíduo, gerando, assim, os impulsos para outras funções, alterando ou mantendo sua tarefa de execução no ato de dirigir (RUEDA; CASTRO, 2010). O processo atencional, assim como o processo de interpretar, selecionar as informações e reagir, tem relação com determinadas diminuições nas habilidades cognitivas do condutor. Essas habilidades são alteradas pelo fator do envelhecimento, variando de indivíduo para indivíduo, porém não de forma a afetar, necessariamente, sua capacidade de condução do veículo (BALBINOT; ZARO; TIMM, 2011).

Considerando essa complexidade, ao dirigir estas funções psicológicas estão envolvidas diretamente na segurança do indivíduo e do trânsito e que levam um indivíduo a realizar uma manobra que seja adequada avaliando os riscos envolvidos. Deve ser observado também como parte da complexidade no ato de dirigir a personalidade de cada condutor, estilos cognitivos, motivação no ato de dirigir e a experiência (BALBINOT; ZARO; TIMM, 2011).

$\mathrm{O}$ envelhecimento tem uma influência importante no desempenho cognitivo ainda na faixa dos 40 aos 50 anos de idade, e não apenas dos 50 aos 80 anos como frequentemente se supõe, com rebaixamento no desempenho de habilidades visuo-espaciais e motoras, mesmo em indivíduos que apresentam escolaridade formal (SALTHOUSE, 2009; SCHROEDER; SALTHOUSE, 2004). Além disso, algumas condições médicas, como doenças neurodegenerativas, podem interferir em funções necessárias para uma direção segura, elevando o risco de acidentes (FERREIRA; SIMÕES, 2015).

Recursos tecnológicos que permitam observar a dinâmica da relação dos comportamentos de condutores e a avaliação das capacidades cognitivas demonstram ser muito importantes. Possibilitam a caracterização de situações de trânsito e consequentemente a interação do condutor com o meio com vias a explorar a capacidade perceptiva e atencional, tomada de decisão e performance (BALBINOT; ZARO; TIMM, 2011; MAYHEW et al., 2011).

No entanto, mesmo na presença de déficits cognitivos, os condutores idosos costumam ser mais experientes no trânsito. A inexperiência de condutores, nesse sentido, também é um dos fatores que levam ao cometimento de erros, 
como é visto em condutores mais jovens, que, apesar da capacidade cognitiva otimizada, estariam sob maior risco de acidentes (KONSTANTOPOULOS; CRUNDALL, 2008). Desta forma, apesar da evidência que o envelhecimento pode trazer prejuízos cognitivos, a experiência em conjunto com outros fatores favorece a escolha de comportamentos compensatórios ao declínio e consequentemente a tomada de decisão (BALDOCK et al., 2006; CHARLTON et al., 2006; DAVIS; CONLON, 2017; FENG et al., 2018; MOLNAR et al., 2013).

\section{Comportamentos de autorregulação, adaptação da personalidade e tomada de decisão em idosos.}

Comportamentos compensatórios de condução ou de autorregulação de condução são considerados um meio pelos quais os motoristas mais velhos podem compensar quedas percebidas na habilidade de dirigir (DAVIS; CONLON, 2017). Diante do envelhecimento, muitos motoristas mais velhos modificam gradualmente ao longo do tempo seu comportamento ao volante para compensar o declínio nas habilidades de condução de um veículo, muitas vezes culminando em decidir parar de dirigir completamente (FENG et al., 2018; HAKAMIES-BLOMQVIST; WAHLSTRÖM, 1998). Exemplos destes comportamentos incluem decisões sobre onde morar ou qual veículo dirigir, assim como comportamentos que evitam situações específicas e que podem ser consideradas desafiadoras, como a redução do tempo de exposição ao volante, dirigir à noite, em áreas desconhecidas, em vias de alta velocidade, além de manter conversação com outros passageiros (MOLNAR et al., 2013). Assim, a autorregulação da direção é composta de diferentes estratégias que ocorrem ao adaptar a tomada de decisão de motoristas mais idosos e que tem uma adequada percepção das quedas cognitivas e perceptivas provocadas pelo envelhecimento (CHARLTON et al., 2006).

De forma geral, o envelhecimento pode ter uma influência importante nas habilidades cognitivas, mas também pode ser que os desempenhos satisfatórios na direção de muitos idosos podem estar fortemente relacionados à quantidade e à qualidade do conhecimento específico adquirido até então (SCHROEDER; SALTHOUSE, 2004). Wikman, Nieminen e Summala (1998) mostraram-se favoráveis à hipótese de ganhos para os motoristas mais velhos relativos à experiência. Acreditam que a experiência para conduzir um veículo é baseada em associações e tempo de aprendizagem de comportamentos automáticos, a fim de que o indivíduo se torne um condutor habilidoso. Esses autores estudaram a interferência do sexo e da experiência de motoristas em tarefas distratoras durante a condução de veículos e concluíram que condutores experientes conseguiram adaptar sua atenção na estrada de forma mais adequada. Eles evitaram manter sua atenção em tarefas secundárias por mais tempo em comparação com motorista menos experientes e assim consequentemente ficaram menos expostos a terem falhas na condução. Certamente os adultos mais velhos se beneficiam da experiência, e isso pode 
ser visualizado no que ocorre com o risco relativo de acidentes em motoristas experientes, que não é tão alto como poderíamos esperar, levando-se em consideração as quedas funcionais comumente experimentadas com a idade (LANGFORD; KOPPEL, 2006).

Alguns aspectos do envelhecimento podem ser favoráveis para a tomada de decisão baseada na autorregulação dos comportamentos ao volante: os aspectos da personalidade (ROBERTS et al., 2007) e a capacidade do processo analítico (ZYSBERG, 2009) são fatores que contribuem para avaliar a previsão de sucesso na tomada de decisão baseadas na experiência de uma pessoa. $\mathrm{O}$ desenvolvimento da personalidade alcança seu ápice na idade adulta, em que os traços de personalidade mostram um padrão claro de mudança normativa ao longo do curso da vida. As pessoas tornam-se mais socialmente dominantes, conscienciosas e emocionalmente estáveis em um processo que se inicia no adulto jovem, mas que aparece mais substancialmente na idade adulta e que tem continuidade na velhice (ROBERTS; WALTON; VIECHTBAUER, 2006). A conscienciosidade prevê maior desempenho e sucesso de vida além de uma capacidade cognitiva mais elevada (JUDGE; KLINGER; SIMON, 2010; ROBERTS et al., 2007). E os indivíduos mais velhos apresentam menos características em fatores de personalidade que expressam ansiedade e busca de emoções (MCADAMS; OLSON, 2010), características estas que são associadas, por exemplo, a comportamentos de risco no trânsito (CORREIA; HORTA, 2014; PANICHI; WAGNER, 2006).

Muitos estudos fornecem evidências de multidirecionalidade, variabilidade e plasticidade na natureza e na direção de mudança na saúde física, funcionamento cognitivo e bem-estar durante o envelhecimento. A otimização dos processos cognitivos, dos traços de personalidade e de fatores sociais seguem um modelo de adaptação como foco principal (BALTES, 1987)metatheoretical view on the nature of development. The family of theoretical perspectives associated with this metatheoretical view of life-span developmental psychology includes the recognition of multidirectionality in ontogenetic change, consideration of both age-connected and disconnected developmental factors, a focus on the dynamic and continuous interplay between growth (gain.

No entanto, de fato nem sempre os idosos desempenham uma capacidade adequada de autorregular seu comportamento (BALDOCK et al., 2006) e isso inclui uma variedade de características pessoais sociais que influenciam os objetivos individuais e consequentemente a tomada de decisão. Alguns motoristas idosos podem atribuir altas classificações ao seu desempenho de condução, mesmo na presença de declínio de habilidades (FREUND et al., 2005). O sexo, a capacidade de auto percepção de habilidade motora e a consciência quanto aos impedimentos funcionais revelam que idosos podem superestimar seu desempenho ou habilidade de condução (BALDOCK et al., 2006; FREUND et al., 2005). 
Um estudo que acompanhou durante 5 anos motoristas idosos relatou que, apesar do aumento de comportamento de autorregulação ao longo do tempo do estudo específico, esses participantes também tiveram duas vezes mais chances de sofrer um acidente por apresentar alguma falha. Demonstraram, assim, que a autorregulação pode ser insuficiente para compensar o risco de acidentes. No entanto, ainda há uma escassez de estudos naturalísticos que relacionem a capacidade cognitiva e o desempenho de condução autorreferida (ROSS et al., 2009).

\section{CONSIDERAÇÕES FINAIS}

Dirigir é uma tarefa complexa que requer um conjunto de habilidades cognitivas (visuais, cognitivas e psicomotoras). À medida que as pessoas envelhecem, experimentarão alguma perda nessas habilidades como resultado do próprio processo de envelhecimento, mesmo que haja uma variação em relação à intensidade com que esses indivíduos experimentam esses declínios. Em um processo de compensação desses declínios, os idosos podem autorregular ou mudar seus comportamentos de condução justamente pela percepção do descompasso entre suas habilidades atuais e o ambiente. Como tal, eles modificam seus comportamentos de condução, evitando certas situações que exigem um maior nível de habilidade. Isso também se deve a uma conjuntura de fatores, como personalidade, nível socioeconômico e educacional e ambiente facilitador. No entanto, não se está aqui apresentando um modelo fiel para a compensação do risco relativo aos acidentes de trânsito baseado em comportamentos autorregulatórios, pois a literatura disponível não identifica o que pode ser aplicado para cada situação específica, como, por exemplo, em casos nos quais há predomínio de um comprometimento cognitivo grave.

Contudo, entendemos que mesmo que muitos idosos modifiquem gradualmente suas escolhas ao conduzir um veículo, o aumento do risco de acidentes não é uma questão da idade em si, mas sim dificuldades funcionais e cognitivas, sendo pertinente que os idosos apresentem a capacidade de por em prática os referidos comportamentos compensatórios. Nesse sentido, algumas modificações na vida diária podem ser necessárias durante o processo de envelhecimento.

Uma mudança natural oriunda do decorrer da vida, que apresenta uma série de consequências negativas, é a perda da mobilidade. Conduzir um veículo, por outro lado, é uma forma de mobilidade muito importante em idosos para a manutenção da sua independência e saúde mental, sendo um suprimento desta falta de mobilidade. Um número de consequências negativas, assim, pode surgir das reduções na capacidade de mobilidade de uma pessoa, pois o ato de dirigir, desacompanhado dos processos autorregulatórios, em vez de ser um suprimento para a falta de mobilidade, pode se tornar um problema maior. 
Com efeito, habilidades psicomotoras e cognitivas são fundamentais para uma direção segura, de acordo com o modelo de comportamento de condução vigente. Entretanto, o risco de colisão de um veículo cuja relação seja diretamente apontada para o envelhecimento ainda não é totalmente conhecido, necessitando de maiores evidências de como se opera a relação entre envelhecimento e desempenho ao volante.

Por outro lado, as teorias da autorregulação de comportamento em motoristas idosos levam às considerações sobre possíveis métodos de treinamento para promover estratégias adaptativas que podem ajudar os condutores mais velhos a manter o desempenho na detecção de perigos quando ao volante e manutenção por um tempo prolongado de mobilidade e independência.

\section{REFERÊNCIAS}

ANSTEY, K. et al. Cognitive, sensory and physical factors enabling driving safety in older adults. Clinical Psychology Review, v. 25, n. 1, p. 45-65, jan. 2005.

BALBINOT, A. B.; ZARO, M. A.; TIMM, M. I. Funções psicológicas e cognitivas presentes no ato de dirigir e sua importância para os motoristas no trânsito. Ciências \& Cognição, v. 16, n. 2, p. 13-29, 2011.

BALDOCK, M. R. J. et al. Self-regulation of driving and its relationship to driving ability among older adults. Accident Analysis \& Prevention, v. 38, n. 5, p. 1038-1045, set. 2006.

BALTES, P. B. Theoretical propositions of life-span developmental psychology: On the dynamics between growth and decline. Developmental Psychology, v. 23, n. 5, p. 611-626, 1987.

BUNCE, D. et al. Age and inconsistency in driving performance. Accident Analysis \& Prevention, v. 49, p. 293-299, nov. 2012.

CASUTT, G. et al. The drive-wise project: driving simulator training increases real driving performance in healthy older drivers. Frontiers in Aging Neuroscience, v. 6, 13 maio 2014.

CHAN, R. et al. Assessment of executive functions: Review of instruments and identification of critical issues. Archives of Clinical Neuropsychology, v. 23, n. 2, p. 201-216, mar. 2008.

CHARLTON, J. L. et al. Characteristics of older drivers who adopt self-regulatory driving behaviours. Transportation Research Part F: Traffic Psychology and Behaviour, v. 9, n. 5, p. 363-373, set. 2006. 
CORREIA, J. P.; HORTA, M. DA P. C. Personalidade e comportamentos de risco de motoristas : diferenças entre sexos. Revista Psicologia: Teoria e Prática, v. 16, n. 1, p. 79-90, 2014.

DAVIS, J. J.; CONLON, E. G. Identifying compensatory driving behavior among older adults using the situational avoidance questionnaire. Journal of Safety Research, v. 63, p. 47-55, dez. 2017.

ENGLE, R. W. Working Memory Capacity as Executive Attention. Society, v. 11, n. 1, p. 19-23, 2002.

FENG, J. et al. Adaptive response criteria in road hazard detection among older drivers. Traffic Injury Prevention, v. 19, n. 2, p. 141-146, 17 fev. 2018.

FERREIRA, I. S.; SIMÕES, M. R. Contributo da avaliação psicológica no exame clínico de condutores com doença neurológica e psiquiátrica: revisão teórica. Revista Portuguesa de Saúde Pública, v. 33, n. 1, p. 57-70, jan. 2015.

FREUND, B. et al. Self-rated driving performance among elderly drivers referred for driving evaluation. Accident Analysis \& Prevention, v. 37, n. 4, p. 613-618, jul. 2005.

GLISKY, E. L. Changes in cognitive function in human aging. In: RIDDLE, D. (Ed.). Brain Aging: Models and Mechanisms. Boca raton: CRC Press/Taylor \& Francis, 2007. v. 21p. 3-20.

\section{GONÇALVES, I. COMPORTAMENTOS DE RISCO: UMA REVISÃO}

BIBLIOGRÁFICA SOBRE AS CAUSAS DE ACIDENTES DE TRÂNSITO NOS

ÚLTIMOS 10 ANOS. Revista Cereus, v. 5, n. 2, 2013.

HAKAMIES-BLOMQVIST, L.; WAHLSTRÖM, B. Why do older drivers give up driving? Accident Analysis \& Prevention, v. 30, n. 3, p. 305-312, maio 1998.

INSTITUTO BRASILEIRO DE PLANEJAMENTO E TRIBUTAÇÃO (IBPT). Histórico da entidade. Curitiba, 2018. Disponível em: <https:/ /ibpt.com.br/noticia/2640/ REAL-FROTA-CIRCULANTE-NO-BRASIL-E-DE-65-8-MILHOES-DE-VEICULOSINDICA-ESTUDO). Acesso em: 10 jul. 2018.

JUDGE, T. A.; KLINGER, R. L.; SIMON, L. S. Time is on my side: Time, general mental ability, human capital, and extrinsic career success. Journal of Applied Psychology, v. 95, n. 1, p. 92-107, 2010.

KAY, L. et al. Validity and reliability of the on-road driving assessment with senior drivers. [s.l: s.n.]. v. 40

KONSTANTOPOULOS, P.; CRUNDALL, D. The Driver Prioritisation Questionnaire: Exploring drivers' self-report visual priorities in a range of driving scenarios. Accident Analysis \& Prevention, v. 40, n. 6, p. 1925-1936, nov. 2008. 
LANGFORD, J.; KOPPEL, S. Epidemiology of older driver crashes - Identifying older driver risk factors and exposure patterns. Transportation Research Part F: Traffic Psychology and Behaviour, v. 9, n. 5, p. 309-321, set. 2006.

MAYHEW, D. R. et al. On-road and simulated driving: Concurrent and discriminant validation. Journal of Safety Research, v. 42, n. 4, p. 267-275, ago. 2011.

MCADAMS, D. P.; OLSON, B. D. Personality Development: Continuity and Change Over the Life Course. Annual Review of Psychology, v. 61, n. 1, p. 517-542, jan. 2010.

MILLER, S. M.; TAYLOR-PILIAE, R. E.; INSEL, K. C. The association of physical activity, cognitive processes and automobile driving ability in older adults: A review of the literature. Geriatric Nursing, v. 37, n. 4, p. 313-320, jul. 2016.

MOGNON, J. F.; SANTOS, A. A. A. Avaliação do comportamento do motorista, lócus de controle e estilos de direção no trânsito. Temas em Psicologia, v. 25, n. 4, p. 16211635, 2017.

MOLNAR, L. J. et al. Tactical, strategic, and life-goal self-regulation of driving by older adults: Development and testing of a questionnaire. Journal of Safety Research, v. 46, p. 107-117, set. 2013.

PANICHI, R. M. D.; WAGNER, A. Comportamento de risco no trânsito: Revisando a literatura sobre as variáveis preditoras da condução perigosa na população juvenil. Interamerican Journal of Psychology, v. 40, n. 2, p. 159-166, 2006.

ROBERTS, B. W. et al. The Power of Personality: The Comparative Validity of Personality Traits, Socioeconomic Status, and Cognitive Ability for Predicting Important Life Outcomes. Perspectives on Psychological Science, v. 2, n. 4, p. 313345, 24 dez. 2007.

ROBERTS, B. W.; WALTON, K. E.; VIECHTBAUER, W. Patterns of mean-level change in personality traits across the life course: A meta-analysis of longitudinal studies.

Psychological Bulletin, v. 132, n. 1, p. 1-25, 2006.

ROSS, L. A. et al. Do Older Drivers At-Risk for Crashes Modify Their Driving Over Time? The Journals of Gerontology Series B: Psychological Sciences and Social Sciences, v. 64B, n. 2, p. 163-170, 1 mar. 2009.

RUEDA, F. J. M.; CASTRO, N. R. I. Capacidade atencional: há decrécimo com o passar da idade?. Psicologia Ciência e Profissão, v. 30, n. 3, p. 572-587, 2010.

SALTHOUSE, T. A. When does age-related cognitive decline begin? Neurobiology of Aging, v. 30, n. 4, p. 507-514, abr. 2009.

SCHROEDER, D. H.; SALTHOUSE, T. A. Age-related effects on cognition between 20 and 50 years of age. Personality and Individual Differences, v. 36, n. 2, p. 393-404, jan. 2004. 
VASQUES, A. M. et al. Desempenho de idosos em simulador de direção e cognição. ConScientiae Saúde, v. 15, n. 4, p. 642-649, 30 dez. 2016.

WATZKE, J.; SMITH, D. B. D. Concern for and knowledge of safety hazards among older people: Implications for research and prevention. Experimental Aging Research, v. 20, n. 3, p. 177-188, 27 jul. 1994.

ZAGORSKY, J. L. Do you have to be smart to be rich? The impact of IQ on wealth, income and financial distress. Intelligence, v. 35, n. 5, p. 489-501, set. 2007.

ZYSBERG, L. An Emerging New Component of Cognitive Abilities in Human Resources Selection: Preliminary evidence to the existence of a 'process-analytic' factor in selection batteries. International Journal of Selection and Assessment, v. 17, n. 1, p. 1-7, 2009. 